\title{
Small-World Rouse Networks as models of cross-linked polymers
}

\author{
S. Jespersen ${ }^{a, b}$ \\ I. M. Sokolov ${ }^{b}$ and A. Blumen ${ }^{b}$ \\ ${ }^{a}$ Institute of Physics and Astronomy \\ University of Aarhus, DK-8000 Arhus C \\ ${ }^{b}$ Theoretische Polymerphysik \\ Universität Freiburg, D-79104 Freiburg i.Br., Germany
}

(October 28, 2018)

We use the recently introduced small-world networks (SWN) to model cross-linked polymers, as an extension of the linear Rouse-chain. We study the SWN-dynamics under the influence of external forces. Our focus is on the structurally and thermally averaged SWN stretching, which we determine both numerically and analytically using a psudo-gap ansatz for the SWN-density of states. The SWN stretching is related to the probability of a random-walker to return to its origin on the $S W N$. We compare our results to the corresponding ones for Cayley trees. 


\section{INTRODUCTION}

Topological properties of polymers can dramatically effect their dynamical properties, such as their collapse in bad solvents and their response to external forcest 1 . Such forces can be applied microscopically, either by having charged polymers (polyelectrolytes, polyampholytes) in electrical fields, or via optical tweezers or magnetic beads 6 . In this communication we study the stretching of cross-linked objects, whose backbones are regular lattices (we will consider for simplicity a ring), a few elements of the backbone being chemically connected to each other via cross-links. An experimental realization may be a very dilute solution of linear chains which are then cross-linked by irradiation $^{\text {or }}$ chemically. Our model is a realization of the so-called small-world networks 10 国 (SWN), and the disorder (the cross-links) is in statistical terms quenched. Considering all bonds to be equal we study the dynamics in the framework of the Rouse model 16.6 , in which the monomers are connected by harmonic springs; we term this structure the smallworld Rouse network (SWRN). Our SWRN is built out of an N-monomer ring with superimposed fixed links between randomly chosen monomers; no additional links are generated or broken in external fields. The SWRN is a new intermediate between linear chains and networks. Distinct from Cayley-trees, which model hyperbranched polymers without loops, loops are a fundamental ingredient here. As such the SWRN is interesting in its own right as a study of the interplay between dynamics and topology, and it belongs to the class of generalized Gaussian structurest $\mathbb{1}^{\mathrm{B}}$. 


\section{DYNAMICS OF SMALL-WORLD ROUSE NETWORKS}

The construction of the SWN which we consider here (see also 1 ), is slightly different from the original one by Watts and Strogat 10 , but preserves its main SWN-characteristics. Starting from a ring of $N$ monomers, we cross-link with probability $p$ each monomer randomly to any of the monomers of the network. Such cross-links thus connect monomers far apart along the chemical backbone (the ring), rendering them close in Eucledian

space. The SWRN-monomers are, in accordance with the Rouse model 16 .67, connected by harmonic springs of strength $k$. The position $\mathbf{R}_{n}(t)$ of the $n$th bead under the action of an external force $\mathbf{F}_{n}(t)$ and in the presence of thermal noise $\boldsymbol{\eta}_{n}(t)$ is governed by the Langevin equation

$$
\gamma \frac{d \mathbf{R}_{n}(t)}{d t}=k \sum_{j=1}^{N} A_{n j} \mathbf{R}_{j}(t)+\mathbf{F}_{n}(t)+\boldsymbol{\eta}_{n}(t) .
$$

Here $\gamma$ is the coefficient of friction, and the matrix $A_{i j}$ is the connectivity matrix of the network. It is defined as follows: Every connection between site $i$ and site $j$ contributes -1 to $A_{i j}$, while $A_{i i}$ and $A_{j j}$ are determined from the condition that $\sum_{j} A_{i j}=\sum_{i} A_{i j}=$ 0. Monasson 2 recently published a detailed study of the spectrum of the small-world connectivity-matrix $A$. Among his findings was the existence of a "pseudo-gap" in the SWN density of states $\rho(E)$ which has the form

$$
\rho(E) \sim E^{-1 / 2} \exp \left(-\frac{C}{\sqrt{E}}\right), \quad E \rightarrow 0
$$

This behavior could not be confirmed numerically through direct diagonlization, and appeared in the data as a real gap. There are, as we shall see, other ways of probing this behavior numerically, and our results support that $\rho(E)$ behaves as Eq. (2). Writing 


$$
\mathbf{R}(t) \equiv\left(\mathbf{R}_{1}(t), \mathbf{R}_{2}(t), \ldots, \mathbf{R}_{N}(t)\right)^{T}
$$

and similary for the other quantities in Eq. (11), we can rewrite the equation of motion in a more condensed form as

$$
\frac{d \mathbf{R}(t)}{d t}=\sigma A \mathbf{R}(t)+\frac{\mathbf{F}(t)}{\gamma}+\mathbf{w}(t)
$$

Here we have introduced $\sigma \equiv k / \gamma$ and $\mathbf{w} \equiv \boldsymbol{\eta} / \gamma$. In the case of a spatially constant external force, the solution to Eq. (4) is obtained as

$$
\mathbf{R}(t)=\int_{-\infty}^{t} d s e^{-\sigma A(t-s)}\left(\frac{\mathbf{F}(s)}{\gamma}+\mathbf{w}(s)\right)
$$

We now specialize to the following situation: the force is switched on at time $t=0$ and pulls only the $m$ th bead in the $y$-direction, i.e. $\mathbf{F}_{i}(t)=\theta(t) \delta_{i, m} F \hat{\mathbf{y}}$. Here $\theta(t)$ is the Heaviside step-function, $\delta_{i, j}$ Kronecker's delta and $\hat{\mathbf{y}}$ is a unit-vector pointing in the $y$ direction. We focus on the displacement of the $m$ th bead along the $y$-axis, and average over thermal noise, using $\langle\mathbf{w}(t)\rangle=0$. Finally we perform a structural average over $m$ and end up with (see e.g.2.

$$
Y(t) \equiv \frac{1}{N} \sum_{m}\left\langle\mathbf{R}_{m, y}(t)\right\rangle=\frac{F}{N \gamma} \int_{0}^{t} d s \sum_{i} e^{-\sigma \lambda_{i} s}=\frac{F t}{N \gamma}+\frac{F}{N \gamma \sigma} \sum_{i=2}^{N} \frac{1-e^{-\sigma \lambda_{i} t}}{\lambda_{i}}
$$

In this equation $\mathbf{R}_{m, y}$ is the $y$ component of $\mathbf{R}_{m}$ and $\lambda_{i}$ with $i=1 \ldots N$ are the eigenvalues of the connectivity matrix $A$. The last expression follows from the fact that for a connected structure, only one eigenvalue vanishes (say, $\lambda_{1}$ ). At times $t$ much smaller than the time scale set by the largest eigenvalue $\lambda_{\max }$, i.e. when $\sigma \lambda_{\max } t \ll 1, Y(t)$ increases linearly in time: $Y(t) \sim F t / \gamma$. That is, only the monomer being pulled moves with a constant speed, not yet feeling the influence of the other monomers. Likewise, at late times $\sigma \lambda_{\min } t \gg 1$, where $\lambda_{\min }$ is the lowest non-vanishing eigenvalue, the entire polymer is being pulled with a constant speed, $Y(t) \sim F t /(N \gamma)$. These observations are independent of the specific 
structure being pulled, and only in the intermediary regime $\lambda_{\text {max }}^{-1} \ll \sigma t \ll \lambda_{\text {min }}^{-1}$ does the particular topology of the polymer affect the dynamics, namely through the spectrum of the connectivity matrix.

The numerical computation of the quantity $Y(t)$ above proceeds as follows. From a specific realization of an $N=1000$ small-world network, we construct the corresponding connectivity matrix. Then we find the $N$ eigenvalues using standard routines, and implement Eq. (6). To get an idea of the importance of sample to sample fluctuations, we consider first 10 different realisations of the SWRN for $p=0.05$. Plotted is in Fig. 11 on double logarithmic scales $Y$ as a function of $t$, where here and in the following we use the dimensionless variables $Y^{*}(t) \equiv \sigma \gamma Y(t) / F$ and $t^{*} \equiv \sigma t$. In Fig. 1 we display the envelope of all 10 realizations, i.e. the two curves are the extremal two "worst" cases. We see that the difference in the results is quite small (and appears, as it should, only for intermediary times), and therefore we regard results from any specific realization as being typical. In Fig. 2 we analyze the dependence of $Y(t)$ on cross-linking, by varying $p$ from $p=0$ (standard Rouse-model of the ring) to $p=0.01$ and $p=0.05$. We note first that the differences are now considerably larger than in Fig. 11. Second, for $p=0$ i.e. for the Rouse chain, we have the standard picture: a subdiffusive $\sqrt{t}$ behavior at intermediary times is followed by a diffusive $t$ behavior at longer times. At very early times we also have a linear behavior, albeit not visible in the range of the figure. The initial and final dynamics are in accordance with the explanation given abovel . The intermediate behavior reflects the structure of the spectrum of $A$, and is also well understood in the Rouse case; it is a result of the rather slow propagation of disturbances through the chain (here the ring).

We infer from the other curves in Fig. 2, that even very small but nonvanishing $p$ affect the intermediate behavior of $Y(t)$ quite strongly. For increasing $p$ the curves bend 
downwards from the $p=0$ case, mirroring the increased stiffness of the polymer due to the additional links. Thus a ring with cross-links can be easily distinguished experimentally (say through NMR or electronic energy transfer) from one without cross-links, whose $Y(t)$ dynamics under $\mathbf{F}$ is never slower than $\sqrt{t}$. As expected, the very early and very late behavior in all three cases coincide, being independent of the specific structure under scrutiny.

In Fig. 3 we plot on logarithmic scales $Y^{*}(t)$ as a function of time $t^{*}$ for several values of $p$. Increasing $p$ increases the stiffness of the polymer, and this is reflected in the intermediary regime, which becomes almost flat for large $p$. Moreover the long time behavior of the polymer is reached much earlier for polymers with a higher number of cross-links. In line with the discussion above of the range of the intermediary regime $\left(\lambda_{\text {max }}^{-1} \ll \sigma t \ll \lambda_{\text {min }}^{-1}\right)$, this feature means that the lowest non-vanishing eigenvalue $\lambda_{\min }$ gets quite large, and it is hence related to the appearance of a (pseudo) gap in the spectrum of $A$.

\section{THEORETICAL ANALYSIS}

As indicated earlier, the initial as well as the asymptotic behavior of $Y(t)$ are well understood. Thus we will concentrate here on the richer and much more complex intermediate behavior. We shall rewrite Eq. (6) using a continuous picture, based on the density of states $\rho(\lambda)=\lim _{N \rightarrow \infty}(1 / N) \sum_{i} \delta\left(\lambda-\lambda_{i}\right)$, but continue to separate out the vanishing eigenvalue from the rest. Hence, with $\epsilon$ very small, $\epsilon \rightarrow 0^{+}$:

$$
Y(t)=\frac{F}{N \gamma} \int_{0}^{t} d s \sum_{i} e^{-\sigma \lambda_{i} s}=\frac{F t}{N \gamma}+\frac{F}{\gamma} \int_{0}^{t} d s \int_{\epsilon}^{\infty} d \lambda \rho(\lambda) e^{-\sigma \lambda s},
$$

an expression which is a fortiori correct in the presence of a gap, where one can take $0<\epsilon \leq \lambda_{\min }$. It will be convenient also to consider the stretching (relative motion) $\Delta(t)$ 
separately:

$$
\Delta(t) \equiv Y(t)-\frac{F t}{N \gamma}=\frac{F}{\gamma} \int_{0}^{t} d s \int_{0^{+}}^{\infty} d \lambda \rho(\lambda) e^{-\sigma \lambda s}
$$

We remark that the inner integral in Eq. (8) is related to the probability for a random walker to be present at the original site1 11, 2 . We therefore first analyse the behavior of this quantity:

$$
P_{0}(t) \equiv \int_{0}^{\infty} d \lambda \rho(\lambda) e^{-\lambda t}
$$

The asymptotic temporal behavior is accessed through the behavior of $\rho(\lambda)$ at small $\lambda$. Inserting the expression of Monasson 12 Eq. (2) into Eq. (9) we obtain:

$$
P_{0}(t) \sim \int_{0}^{\infty} d \lambda \lambda^{-1 / 2} \exp \left(-\frac{C}{\sqrt{\lambda}}-\lambda t\right)=-t^{-2 / 3} \frac{d}{d C} \int_{0}^{\infty} d y \exp \left(-t^{1 / 3}\left(\frac{C}{\sqrt{y}}-y\right)\right)
$$

The asymptotic behavior of the integral follows readily from a saddle-point procedure 19 , so that we end up with

$$
P_{0}(t) \sim t^{-1 / 2} \exp \left(-C^{\prime} t^{1 / 3}\right)
$$

whith $C^{\prime}=3(C / 2)^{2 / 3}$. This is by itself a quite interesting and novel result, and it compares favourably to our numerical simulations 11 for $P_{0}(t)$. The result is close in form to that for Cayley trees2021, where $P_{0}(t) \sim t^{-3 / 2} \exp (-c t)$. Inserting Eq. (11) in Eq. (8) and reintroducing $\sigma$, we get

$$
\Delta(t) \sim \frac{3 F}{C^{\prime} \gamma \sigma}\left(\frac{1}{2} \sqrt{\frac{\pi}{C^{\prime}}}-(\sigma t)^{1 / 6} e^{-C^{\prime}(\sigma t)^{1 / 3}}\right)
$$

Notice that for very large $t$ the stretching $\Delta(t)$ of the SWRN tends to a constant $\Delta_{\infty}$. In the units of our figure this constant depends mainly on $C^{\prime}$ (since $F, \gamma$ and $\sigma$ drop out); theoretically one may obtain $C^{\prime}$ and also $C$ out of $\Delta_{\infty}$.

In Fig. 4 we plot the dimensionless stretching $\Delta^{*}(t) \equiv Y^{*}(t)-\sigma t / N$ for $p=0.05$ and compare it to the analytical form Eq. (12). We do this by fitting $a-b t^{1 / 6} \exp \left(-c t^{1 / 3}\right)$ 
to the data, and as can be inferred from Fig. 4, the agreement is very convincing. From the least-squares fit we obtain $a=5.09, b=7.67$ and $c=0.54$. We remark that the agreement at short times may be rendered even better by also keeping the next term in the expansion of the integral of $P_{0}(t)$, a term which is proportional to $t^{-1 / 6} \exp \left(-C^{\prime} t^{1 / 3}\right)$. Furthermore we remark that for Cayley-trees the intermediate behavior of $Y(t)$ can also be determined in a similar manner: The saddle-point approach yields to leading order:

$$
Y(t) \sim \tilde{a}-\tilde{b} t^{-3 / 2} \exp (-\tilde{c} t)
$$

with $\tilde{a}, \tilde{b}$ and $\tilde{c}$ being constants.

\section{CONCLUSIONS}

In this communication we have studied the behavior of a small-world network model (the SWRN) of a linked ring-polymer, focusing on its dynamics under external forces. Thus the motion of a monomer pulled by such a force is vastly different, depending on whether the monomer belongs to a SWRN or to a simple ring without cross links. This may enable via NMR or electronic energy transfer22 to distinguish clearly between crosslinked and non-cross-linked polymers. Our numerical results for the stretching of the SWRN under external forces are in excellent agreement with our analytical expressions, which used the pseudo-gap behavior of the SWN density of states, as postulated in former work 12. As discussed in the present communication, these results are directly connected to expressions for the return to the origin of a random walker on the SWN. 


\section{ACKNOWLEDGMENTS}

The support of the DFG, of the GIF through grant I0423-061.14, and of the Fonds der Chemischen Industrie are gratefully acknowledged. 
${ }^{1}$ J.-U. Sommer and A. Blumen, J. Phys. A 28, 6669 (1995).

${ }^{2}$ H. Schiessel, G. Oshanin and A. Blumen, Macromol. Theory Simul. 5, 45 (1996).

${ }^{3}$ H. Schiessel, Phys. Rev. E 57 5, 5775 (1998).

${ }^{4}$ P. Biswas, R. Kant and A. Blumen, Macromol. Theory Simul. 9, 56 (2000).

${ }^{5}$ Y. Kantor and M. Kardar, Phys. Rev. E 54 5, 5263 (1996).

${ }^{6}$ S. R. Quake, H. Babcock and S. Chu, Nature 388, 151 (1997).

${ }^{7}$ T. T. Perkins, D. E. Smith, R. G. Larson and S. Chu, Science 286, 83 (1995).

${ }^{8}$ D. Wirtz, Phys. Rev. Lett 75, 2436 (1995).

${ }^{9}$ S. Klaumünzer, Q. Q. Zhu, W. Schnabel, G. Schumacher, Nucl. Instr. and Meth. B 116, 154 (1996), and references therein.

${ }^{10}$ D. J. Watts and S. H. Strogatz, Nature 393440 (1998).

${ }^{11}$ S. Jespersen, I. M. Sokolov, A. Blumen, submitted to Phys. Rev. E.

${ }^{12}$ R. Monasson, Eur. Phys. J. B 12, 555 (2000).

${ }^{13}$ M. E. J. Newman and D. J. Watts, Phys. Rev. E 60 6, 7332 (1999).

${ }^{14}$ C. F. Moukarzel, Phys. Rev. E. 60 6, R6263 (1999).

${ }^{15}$ M. E. J. Newman and D. J.Watts, Phys. Lett. A 263, 341-346 (1999).

${ }^{16}$ P. E. Rouse, J. Chem. Phys. 21, 1272 (1953).

${ }^{17}$ M. Doi and S. F. Edwards, The theory of Polymer Dynamics, Clarendon Press (1986).

${ }^{18}$ J.-P. Bouchaud and A. Georges, Phys. Rep. 195, 12 (1990).

${ }^{19}$ N. G. De Bruijn, Asymptotic Methods in Analysis, Dover Publications (1958).

${ }^{20}$ D. Cassi, Phys. Rev. B 45 1, 454 (1992). 
${ }^{21}$ D. Cassi, Europhys. Lett. 9, 627 (1989).

${ }^{22}$ A. Blumen, J. Klafter and G. Zumofen, in Optical Spectroscopy of Glasses, I. Zschokke ed., p. 199 (Reidel, Dordrecht, 1986). 


\section{FIGURES}

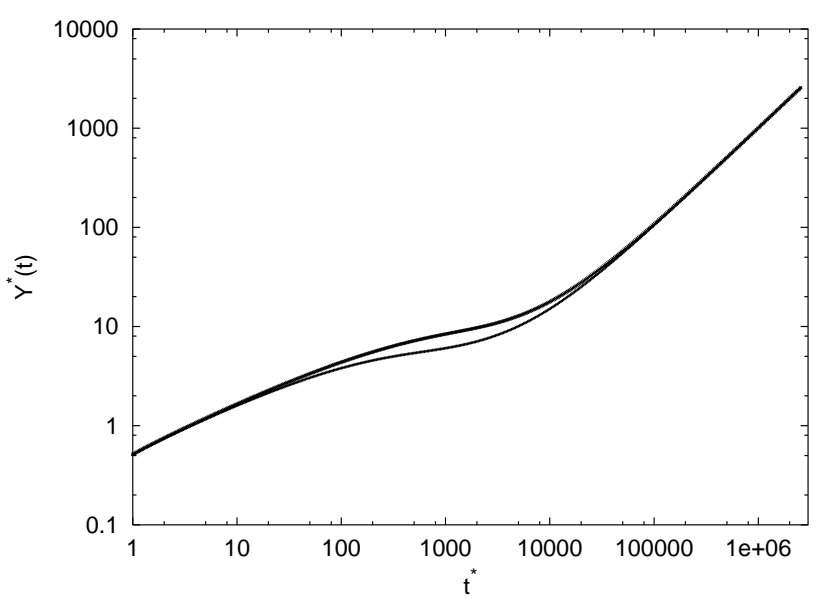

FIG. 1.

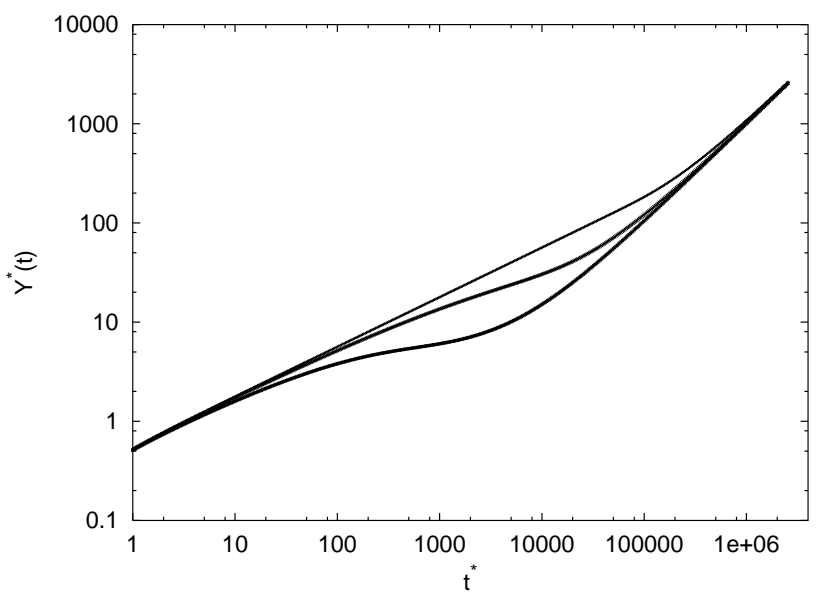

FIG. 2.

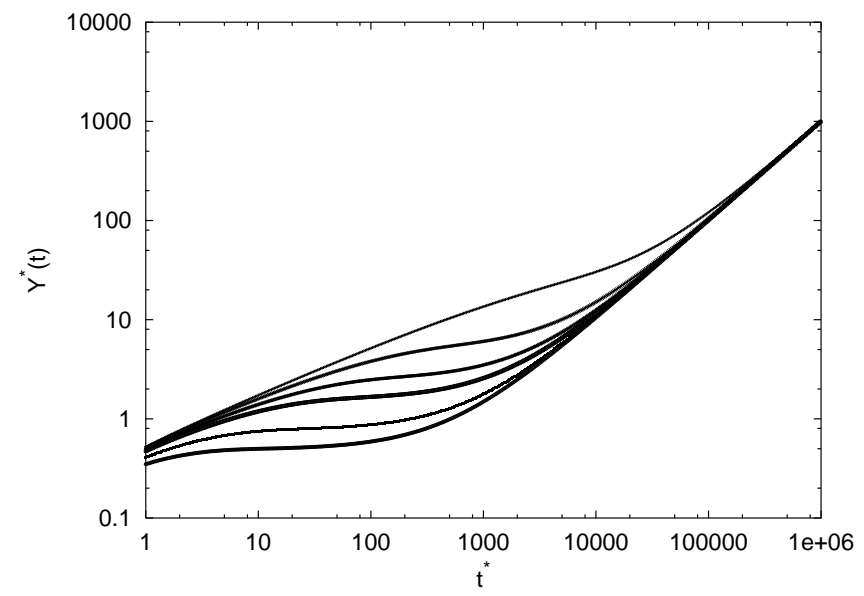

FIG. 3. 


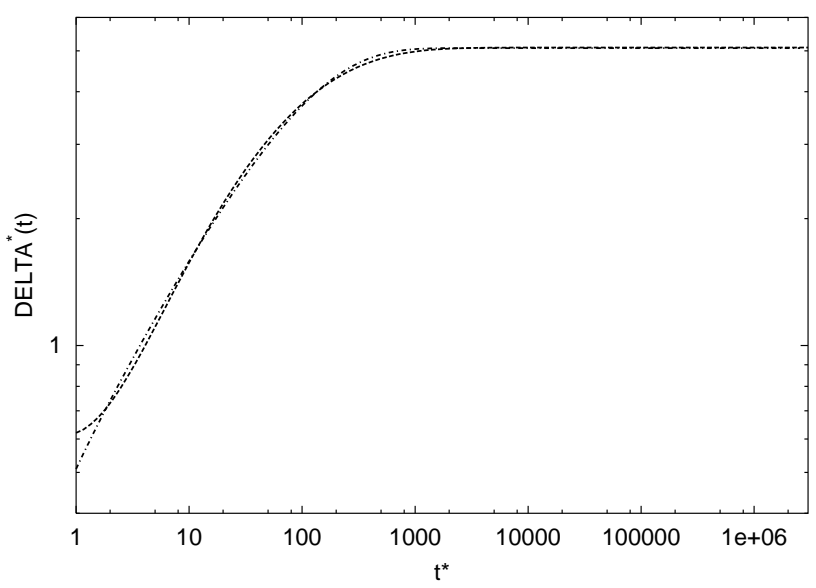

FIG. 4. 


\section{CAPTIONS}

FIG. 1. Two different realizations give rise to similar behavior of $Y(t)$, here plotted on logarithmic-logarithmic scales for $p=0.05$.

FIG. 2. On double logarithmic scales we plot the position $Y(t)$ as a function of time. From upper to lower curve, $p=0, p=0.01$ and $p=0.05$.

FIG. 3. On double logarithmic scales we plot the position $Y(t)$ as a function of time. From upper to lower curve, $p=0.01, p=0.05, p=0.1, p=0.2, p=0.5$ and $p=0.8$.

FIG. 4. Comparison of the theoretical prediction (dashed) with the data (dash-dotted), for $p=0.05$. 